\section{Thermal Conduction and Gas Analysis}

GAS analysis based on thermal conduction was first introduced by Daynes and Shakespear ${ }^{\perp}$ and depends on the principle that the thermal conduction loss in a particular mixture depends uniquely upon the composition of the mixture, and consequently, if we know the conductivity, the composition can be determined. Since then this principle has been utilized by a number of workers to analyse both the steady state and also the approach to steady state of non-uniform gas mixtures under various conditions. Unfortunately, the procedure adopted has not always been free from objections, and in the present report I propose to discuss the use of a thermal conductivity apparatus for which the theory is now completely understood and the various corrections are exactly calculable. The general design of the thermal conductivity cell is based on the conventional hot-wire type of cell introduced by Kannuluik and Martin ${ }^{2}$ and since then has been widely used by a number of workers. Two simple designs for the construction of such colls have been recently made by Srivastava and Saxena ${ }^{3}$.

Until now most workers have used this thermal conductivity apparatus moro or less in an empirical fashion and obtained calibration curves in terms of galvanometer deflexions against concentra. tion when the same current is passed through the helix of the conductivity cell. This procedure involves several discrepancies as the galvanometer deflexion does not give directly the thermal conductivity, though the latter depends uniquely upon the composition alone. This calibration for a particular gas mixture is correct only at the actual experimental points, and any interpolation is subject to errors arising out of the different accommodation coefficients, wall effects and convection effects with varying composition of the mixture unless the katharometer is so designed as to eliminate or correct precisely for the above effects. The theory of the hot-wire cell using a thick wire has been given by Kannuluik and Martin ${ }^{2}$ and seems now to be complete. The corrections to be usually applied in a hot-wire type of apparatus are: $(a)$ convection effects; $(b)$ wall effects; (c) radiation correction; (d) radial flow correction; (e) the temperature jump correction. The conductivity cells used by us $^{3}$ were found to be free from convection and had a negligible temperature jump effect for a considerable range of pressures $(6-20 \mathrm{~cm}$. of mercury). We have therefore talken the observations at a pressure of $10 \mathrm{~cm}$. of mercury. For our metal body cells, calculations showed that the wall effect is of the order of 0.01 per cent for gases like helium and neon while for gases of low thermal conductivity like argon, krypton and xenon, etc., the correction is negligible. The correction due to radiation amounts to the reduction in the conductivity value by a fixed amount and is independent of the nature and composition of the gases. The radial flow correction for a given cell and for a fixed value of the current uniquely depends upon the value of the conductivity and therefore upon $\left(R-R_{0}\right.$ ) approximately. Here $R$ represents the resistance of the cell wire for a certain current $I$, and $R_{0}$ for zero current.

In view of the above remarks about the corrections to be applied to the conductivity values measured by a hot-wire cell, it follows from the theory of Kannuluik and Martin ${ }^{2}$ that for a given mixture the change in resistance $R-R_{0}$ of the cell wire will

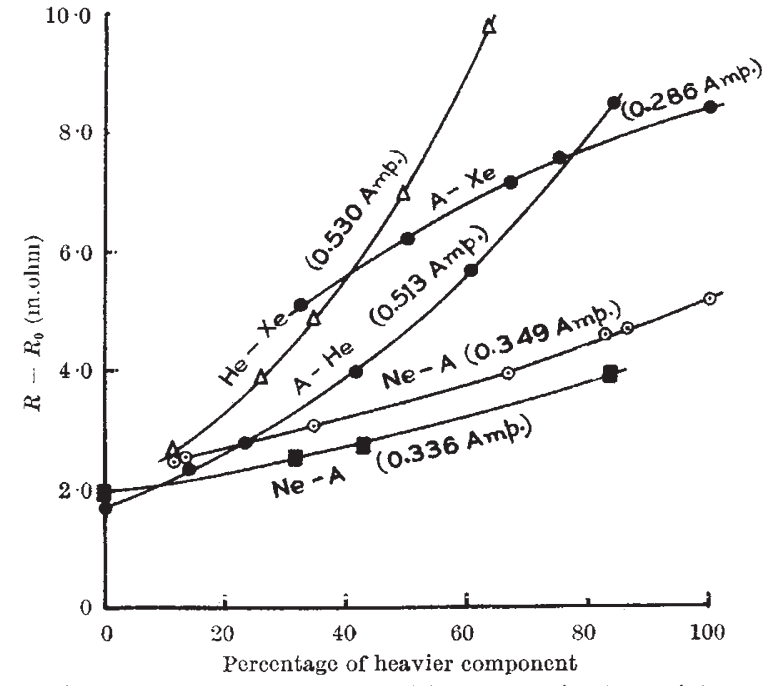

Fig. 1. Plots of $R-R_{0}$ vs. composition for some inert gas mixtures

depend uniquely upon the composition of the mixture provided the same current is passed through the cell wire. Consequently, if we first perform an experiment with a mixture of known composition and plot a graph of $R-R_{0}$ versus composition of the mixture for practically a fixed value of the current, then we can use these plots to find the unknown composition from a knowledge of $R-R_{0}$ only.

Srivastava and Saxena ${ }^{3}$ and Saxena ${ }^{4}$ have recently obtained results of this type for a number of inert gas mixtures and utilized it to calculate the thermal conductivity. In Fig. 1 are shown the plots of $R-R_{0}$ versus composition for some mixtures. The experimental currents to which the data refer are recorded for each curve within brackets. These plots can now be utilized with fair accuracy for the purpose of gas analysis.

I am grateful to Prof. B. N. Srivastava for his kind interest and supervision throughout the progress of the work presented here and to the Council of Scientific and Industrial Research, New Delhi, for financial support.

$$
\text { S. C. Saxena }
$$

Department of General Physics and X-Rays, Indian Association for the Cultivation of Science, Calcutta 32. Oct. 15.

${ }^{1}$ Daynes, H. A., and Shakespear, G. A., Proc. Roy. Soc., A, 97, 273 (1920).

${ }^{2}$ Kannuluik, W. G., and Martin, L. H., Proc. Roy. Soc., A, 144, 496 (1934).

${ }^{3}$ Srivastava, B. N., and Saxena, S. C., Proc. Phys. Soc. (in the press). ${ }^{4}$ Saxena, S. C. (in course of publication).

\section{Free Radical Salts of Halogenated Quinones}

It has been shown ${ }^{1}$ that bi-radical molecular compounds can be formed from quinones $(\mathrm{O}-\mathrm{Q}-\mathrm{O})$ and aromatic diamines $(D)$, and that these compounds are of the ionic form $\mathrm{O}-Q-\mathrm{O}] \cdot D^{+}$.

Highly electropositive aromatic diamines can form stable solid paramagnetic salts with simple anions ${ }^{2}$, for example, the perchlorate of Wurster's ion, $\left.\left(\mathrm{CH}_{3}\right)_{2} \mathrm{~N}-\longrightarrow-\mathrm{N}\left(\mathrm{CH}_{3}\right)_{2}\right]^{+} \mathrm{ClO}_{4}^{-}$, and it was interesting to see if there also existed stable salts of quinones with simple cations : 